\title{
Screening of the Behavioral Tests for Monitoring Agonistic Behavior of Layer Chicks
}

\author{
Said Majdood Raihan ${ }^{1}$, Masaoki Tsudzuki ${ }^{1,2,3}$ and Shin-Ichi Kawakami ${ }^{1,2,3}$ \\ ${ }^{1}$ Graduate School of Biosphere Science, Hiroshima University, Higashi-Hiroshima, Hiroshima 739-8528, Japan \\ 2 Japanese Avian Bioresource Project Research Center, Hiroshima University, Higashi-Hiroshima, Hiroshima 739-8528, Japan \\ ${ }^{3}$ The Research Center for Animal Science, Hiroshima University, Higashi-Hiroshima, Hiroshima 739-8528, Japan
}

\begin{abstract}
Chicken agonistic behavior, a type of social behavior related to threatening and fighting, is among the most serious problems in the poultry industry. However, due to luck of effective models for investigating the brain mechanisms of the behavior, no effective measures have been taken. This study, therefore, aimed to select the behavioral tests available for monitoring chicken agonistic behavior. Two behavioral tests, resident-intruder (R-I) test and social interaction (SI) test, were performed for 10 minutes in 10 pairs of male layer chicks at 8, 12, 16, 20, and 24 days of age, and total agonistic frequencies (TAF: Sum of the frequencies of agonistic displays like pecking, biting, kicking, threatening, and leaping) and latency (the period of time from the beginning of the behavioral test to the occurrence of the first agonistic behavior) were measured as indices of agonistic behavior. Two-way repeated measures ANOVA revealed significant differences in TAF and latency between aggressors and opponents in both the behavioral tests. In the R-I test, the TAF of aggressors significantly increased from 8 to 20 days of age, and the latency significantly decreased from 8 to 24 days of age. In the SI test, however, the TAF of aggressors significantly increased and the latency significantly decreased only from 16 to 20 days of age. When the criterion of high agonistic behavior was defined as the TAF, where aggressors showed more than 30 times of TAF and the opponents did less than one-third TAF of aggressors, the aggression establishment rate (AER), which is equal to the number of aggressors showing high agonistic behavior per total behavioral trials, was significantly higher in the R-I test than in the SI test. These results suggest that the R-I test, rather than the SI test, is an effective tool for monitoring agonistic behavior of layer chicks.
\end{abstract}

Key words: agonistic behavior, layer chicks, resident-intruder test, social interaction test

J. Poult. Sci., 54: 296-302, 2017

\section{Introduction}

Agonistic behavior, as shown by threatening and fighting, is normally directed toward another individual of the same species (Nelson and Trainor, 2007). It is supposed to be an influential element in group communication, with the intention of access to resources that are in limited supply, such as feed, mates, and territory (Blanchard and Blanchard, 2003). Agonistic behavior is also used to form a social dominance hierarchy in a group, which is thought to lead a dominant animal to gain access to these resources (Issa et al., 1999) and reduce unnecessary conflict and risk of injury (Miczek et al., 2001).

Traditionally, the study of agonistic behavior has been conducted in laboratory rodent models. The importance of

Received: February 8, 2017, Accepted: April 4, 2017

Released Online Advance Publication: April 25, 2017

Correspondence: Dr. Shin-Ichi Kawakami, Graduate School of Biosphere

Science, Hiroshima University, Higashi-Hiroshima, Hiroshima 739-8528,

Japan. (E-mail: skawak@hiroshima-u.ac.jp) male hormones, such as testosterone, in stimulating agonistic behavior was studied by Beeman (1947), who found that castration resulted in the failure of male mice to show agonistic behavior, and subcutaneous treatment of testosterone propionate restored the display of agonistic behavior in the castrated mice. Neurotransmitters such as serotonin (5HT) are also known to be a prominent regulator of agonistic behavior. Central administration of 5-HT1A and $1 \mathrm{~B}$ receptor agonists suppressed agonistic behavior in rats (de Boer and Koolhaas, 2005), and mutant mice lacking 5-HT1B receptor showed greater agonistic behavior (Saudou et al., 1994). Agonistic behavior is reported to be elicited from various regions throughout the brain, and the hypothalamus is one of the most prominent areas associated with agonistic behavior (Bernhardt, 1997). Previous studies showed that electrical stimulation of the hypothalamus induced violent attacks in rats (Panksepp, 1971), and immunostaining of c-Fos, as a marker of neuronal activation, revealed that aggression resulted in activation of various nuclei of the hypothalamus of rodents (Kollack-Walker and Newman, 1995; Martinez et 
al., 1998; Haller et al., 2006). A recent study also showed that the optogenetic stimulation of a specific hypothalamic nucleus, named ventrolateral subdivision of the ventromedial hypothalamus, induced agonistic behavior in residents toward intruders, and optogenetic silencing of the neurons in this nucleus reversibly suppressed agonistic behavior (Lin et al., 2011). These researches of rodent aggression biology, as mentioned above, have provided insights for understanding the neurobiological and molecular systems that mediate agonistic behavior. However, the mechanisms of agonistic behavior in other species, such as chickens, remain unknown.

Chickens are social animals, living naturally in groups which are commonly formed with a stable social structure (McBride et al., 1969). Environmental factors including high rearing density, changes in group membership, isolation, and exposure to intruders are known to induce an intense and prolonged distress in birds (Jones and Harvey, 1987), which ultimately results in the induction of agonistic behavior (Al-Rawi and Craig, 1975). Although aggression is a normal display of an instinct in almost all animals, excessive aggression leads to serious economic problems in poultry farms: Male broilers are reported to display high levels of aggression against female conspecifics, which results in reduced fertility and increased mortality of the female chickens (Millman et al., 2000). Moreover, aggression not only reduces productivity, but also results in injury and stress in the chickens, which have been known to be profound issues for animal welfare (Hester, 2005). It is, therefore, of great importance to understand the mechanisms of chicken agonistic behavior and find a way to control excessive aggression of chickens in the poultry industry.

To understand the mechanisms regulating chicken agonistic behavior, it is necessary in the first step to establish effective behavioral models to quantitatively estimate agonistic behavior of the chicken. In general, two behavioral models have been used to assess agonistic behavior of male animals. The first model is isolation-induced aggression, which can be monitored by a kind of behavioral test, named social interaction (SI) test (Silverman et al., 2010). The SI test is a kind of open-field test to study sociality between a pair of animals. Two animals are simultaneously transferred to the diagonal corners of the cage, and various social behaviors like sniffing, grooming, tail-rattling, and attacking are monitored. To monitor agonistic behavior of animals by the SI test, an isolation-housed male animal is made to encounter a group-housed male in an unfamiliar test cage and agonistic behavior of both the animals is monitored. The SI test is thought to have high experimental reproducibility of agonistic behavior of animals because it utilizes social isolation to induce agonistic behavior. Social isolation is reported to induce depression- and anxiety-like symptoms, and these consequently increase aggression (Matsumoto et al., 2005). The second model is the territorial aggression, which can be monitored by another behavioral test, named resident-intruder (R-I) test (Miczek et al., 2001). The R-I test can be used to detect the degree of territorial aggressiveness induced by intrusion of another animal. In the R-I test, a male animal (resident) is reared in isolation in its home cage for more than 1 week. Then, another male conspecific (intruder), which has been reared in a group, is put in the cage, and agonistic behavior of the resident or intruder is monitored. In the R-I test, the resident is thought to display agonistic behavior because it establishes a territory during social isolation in its home cage and attempts to protect the area according to its territoriality (Koolhaas et al., 2013). It could be possible, therefore, to estimate agonistic behavior of chickens by the 2 behavioral tests, as mentioned above. However, there are no previous reports showing whether either or both of the tests are suitable to assess agonistic behavior of chickens.

The purpose of this study was, therefore, to select the behavioral tests available for monitoring chicken agonistic behavior. To monitor agonistic behavior male layer chicks were used in the present study, because they are available as an experimental animal model of the research of agonistic behavior with little expenditure of money, and the research results from them are thought to contribute to solve the problems of aggression by breeder male chickens toward females.

\section{Materials and Methods}

\section{Animals}

Day-old male layer chicks (Julia Lite) were obtained from a local hatchery (Akita Co., Ltd., Hiroshima, Japan). The chicks were maintained in a room $(3.4 \times 3.5 \times 2.1 \mathrm{~m}$, length $\times$ width $\times$ height) with 20 -h lighting and 4-h dark with lights on at $3 \mathrm{AM}$, and with temperature set at $30^{\circ} \mathrm{C}$. They were given free access to a commercial starter diet (Chubushiryo Co., Ltd., Aichi, Japan), and water during the experimental period. The chicks were reared in groups ( 4 chicks per cage) until 2 days of age in the home cages $(30 \times 20 \times 25 \mathrm{~cm}$, length $\times$ width $\times$ height). From 3 days of age, the chicks were divided into 2 groups, isolated-raising ( 1 chick per cage, as aggressors) and grouped-raising ( 3 or 4 chicks per cage, as opponents), and reared in the home cages up to the time of the experiments. All experimental protocols were approved by the Animal Experiment Committee of Hiroshima University.

\section{Social Interaction (SI) Test}

The SI test was performed in 10 pairs of male layer chicks at $8,12,16,20$, and 24 days of age, as described by Kjaer and Jørgensen (2011), with some modification. After measuring body weight with an electronic scale (HF-2000, A\&D Company Ltd., Tokyo, Japan), pairs of chicks from isolated(aggressor) and group-raised (opponent) were simultaneously transferred by hand to the diagonal corners of the transparent acrylic cage $(28 \times 20 \times 20 \mathrm{~cm}$, length $\times$ width $\times$ height), and agonistic behavior of the aggressor and the opponent was recorded manually for 10 minutes by 2 observers who were located more than 1 meter apart from the cage. For the indices of agonistic behavior of the chicks, TAF and latency were determined. TAF is defined as the sum of the frequencies of pecking, biting, kicking, threatening, and leaping. The percentage of each agonistic display in the test was 
also determined as the frequency of each agonistic display per those of the total agonistic displays. Brief descriptions of each agonistic display are as follows (Xie et al., 2010): pecking: the male chick pecks the opponent's body or head; biting: the male chick bites the opponent's body, head, or legs; kicking: the male chick kicks the opponent's body; threatening: the male chick stands in front of another male with its neck and head raised and wings slightly extended; and leaping: the male chick jumps toward his opponent while the opponent flees. Latency is defined as the period of time $(\mathrm{sec})$ from the beginning of the behavioral test to the occurrence of the first agonistic behavior (Xie et al., 2010). All the tests were conducted between 9 AM and 1 PM.

\section{Resident-intruder (R-I) Test}

The R-I test was performed on 10 pairs of male layer chicks at $8,12,16,20$, and 24 days of age, with some modification of the previous report of mice (Miczek et al., 2001). After measuring the body weight, a group-raised (opponent) chick was transferred to the home cage where a chick of isolated-raising was reared. Agonistic behaviors of the aggressor and the opponent were recorded for 10 minutes, and TAF, latency, and the percentage of each agonistic display were determined as described above. All the tests were conducted between $9 \mathrm{AM}$ and $1 \mathrm{PM}$.

\section{Aggression Establishment Rate (AER)}

To compare aggressiveness of the chicks between SI and R-I tests, we calculated the AER, which is equal to the number of aggressors showing high agonistic behavior per total behavioral trials. The criterion of high agonistic behavior was defined as the TAF, where aggressors showed more than 30 times of TAF and the opponents did less than one-third TAF of aggressors. The formula means that the AER is defined as a rate of aggressors showing high aggressiveness with few counterattacks from opponents.

\section{Statistical Analyses}

For comparisons of the body weight, TAF, and latency between aggressors and opponents, we performed two-way repeated measures analysis of variance (ANOVA) with MIXED procedure of SAS for Windows software version 9.4
(SAS Institute Inc., Cary, NC, USA). The statistical model included fixed effects for treatment, day, and treatment $X$ day interaction, with chick as a random effect. For the comparison of the percentage of each agonistic display, we performed two-way ANOVA with SAS. The significance of the differences between means was assessed using a TukeyKramer test. For the comparison of the AERs between SI and R-I tests, the AERs in each test during the whole experimental period were pooled and analyzed using Pearson's chi-square test with FREQ procedure of SAS. Statistical significance was set as $P<0.05$.

\section{Results}

Two-way repeated measures ANOVA revealed that there were no significant differences of body weight between the aggressors and opponents in both behavioral tests (data not shown). The TAF of the aggressors in both tests significantly increased (in the R-I test: $P<0.0001$; in the SI test: $P$ $<0.0001$ ) and their latency significantly decreased (in the RI test: $P<0.0001$; in the SI test: $P<0.0001$ ), as compared to those of the opponents. The effect of day was significant on the TAF of the R-I test $(P<0.01)$ and on latency of SI test $(P$ $<0.01)$. A significant interaction of treatment $\times$ day was observed on the TAF of R-I test $(P<0.01)$ and of SI test $(P<$ $0.05)$.

In the R-I test, the TAF of the aggressors significantly increased from 8 to 20 days of age (Fig. 1b), and their latency significantly decreased from 8 to 24 days of age (Fig. $2 b$ ). In the SI test, however, the TAF of the aggressors significantly increased and their latency significantly decreased only from 16 to 20 days of age (Fig. 1a, 2a).

Table 1 shows the percentage of each agonistic display in both the behavioral tests. Pecking and biting were observed in both tests, but kicking and threatening were observed only in the SI test. Leaping was not observed in both the tests. Two-way ANOVA revealed that the percentage of aggressors' pecking in SI test was significantly lower than those of the others. The percentage of aggressors' biting was significantly higher than that of the opponents in both the tests,

Table 1. The percentage of each agonistic display of aggressors and opponents in the resident-intruder (R-I) and social interaction (SI) tests. The percentages of each agonistic display in the tests were determined as the frequency of each agonistic display per those of the total agonistic displays

\begin{tabular}{lccccc}
\hline \hline & \multicolumn{4}{c}{ Behavioral frequencies (\%) } \\
\cline { 2 - 3 } \cline { 5 - 6 } \cline { 5 - 6 } Behavioral displays & $\begin{array}{c}\text { Resident } \\
\text { (aggressor) }\end{array}$ & $\begin{array}{c}\text { Intruder } \\
\text { (opponent) }\end{array}$ & & $\begin{array}{c}\text { Isolated } \\
\text { (aggressor) }\end{array}$ & $\begin{array}{c}\text { Srouped } \\
\text { (opponent) }\end{array}$ \\
\hline Pecking & $90.6 \pm 1.05^{\mathrm{a}}$ & $89.6 \pm 9.96^{\mathrm{a}}$ & & $78.8 \pm 1.30^{\mathrm{b}}$ & $89.6 \pm 4.64^{\mathrm{a}}$ \\
Biting & $9.41 \pm 1.05^{\mathrm{b}}$ & $0.43 \pm 0.43^{\mathrm{cd}}$ & & $18.6 \pm 1.20^{\mathrm{a}}$ & $2.44 \pm 0.46^{\mathrm{cd}}$ \\
Kicking & 0 & 0 & & $1.34 \pm 0.37$ & $6.08 \pm 3.52$ \\
Threatening & 0 & 0 & & $1.24 \pm 0.26$ & $1.84 \pm 1.14$ \\
Leaping & 0 & 0 & 0 & 0 \\
\hline
\end{tabular}

Values are means \pm SEM. Different letters within rows indicate significant differences $(P<0.05)$. 

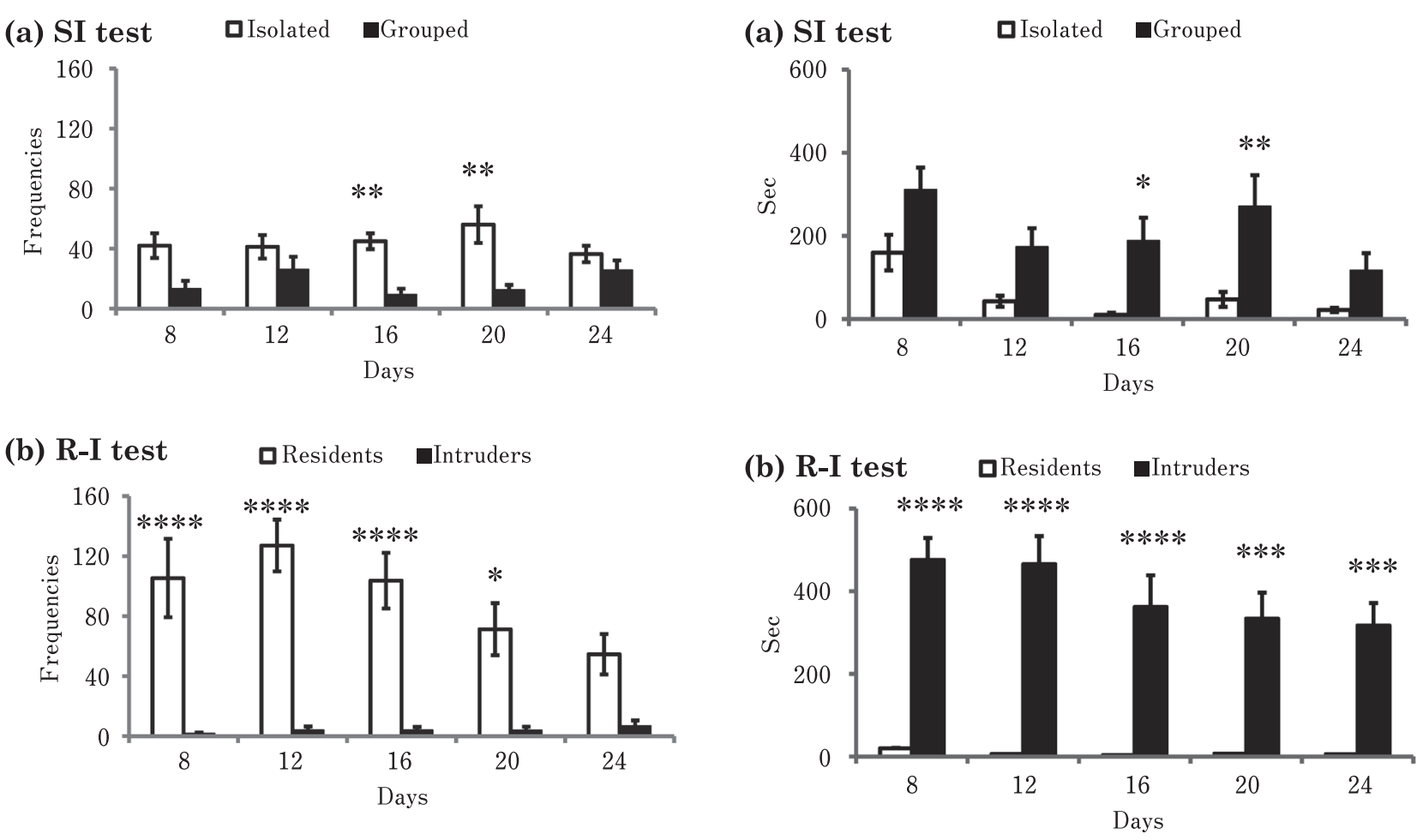

Fig. 1. Total agonistic frequencies (TAF) of aggressors (open column) and opponents (closed column) in the (a) SI and (b) R-I tests. TAF is defined as the sum of the frequencies of pecking, biting, kicking, threatening, and leaping. *: $P<0.05$; **: $P<0.01$; ****: $P<0.0001$, compared with opponents.

and the percentage of the aggressors' biting in SI test was significantly higher than that in R-I test.

Pearson's chi-square test revealed that the AER was significantly higher in the R-I test than in the SI test $(P<$ 0.0001, Fig. 3).

\section{Discussion}

In the present study, the TAF and latency of the aggressors in both SI and R-I tests were significantly different as compared to those of the opponents (Fig. 1 and 2). As TAF and latency are thought to be good indices for estimating aggressiveness of animals, it is suggested that both behavioral tests can be used to monitor agonistic behavior of chicks. However, when AER (defined as the rate of aggressors showing high aggressiveness with few counterattacks from opponents) was used to compare aggressiveness of the chicks as assessed by the 2 behavioral tests, AER was significantly higher in the R-I test than in the SI test (Fig. 3). Since AER is a new index to estimate the aggressiveness of animals by considering the TAF of both aggressors and opponents more accurately, the present results suggest that the R-I test, rather than the SI test, is an effective tool for monitoring agonistic behavior of layer chicks. The R-I test is known to be a behavioral paradigm which stimulates agonistic behavior of territorial animals (Koolhaas et al., 2013). One of the ani-

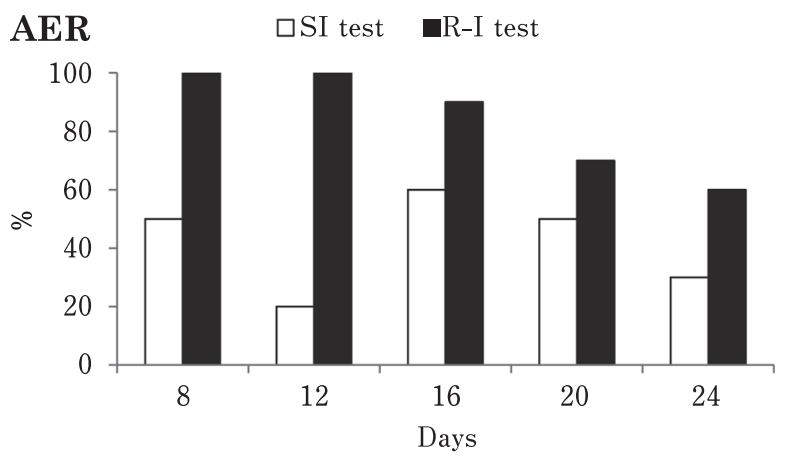

Fig. 3. Aggression establishment rate (AER) in the SI and R-I tests. AER equals the number of aggressors showing high agonistic behavior per that of total behavioral trials. The criterion of high agonistic behavior was defined as the TAF, where aggressors showed more than 30 times of TAF and the opponents did less than one-third TAF of aggressors.

mals (the resident) is allowed to establish a territory in its home cage and another conspecific animal (the intruder) is placed into the resident's home cage, and researchers observe 
the spontaneous and natural expression of both offensive aggression of the resident and defensive behavior of the intruder in a semi natural laboratory setting. Since chickens are territorial animals (McBride and Foenander, 1962) and two chicks in the SI test were placed in an unfamiliar cage where neither had established territory during behavior trials, it was easier to induce agonistic behavior of the chicks in the R-I test than in the SI test.

The present results revealed that the isolated chicks showed more agonistic behavior than the grouped ones, following the R-I and the SI tests series conducted from 8 to 24 days of age (Fig. 1 and 2). Consistent with our results, previous reports also showed that chicks reared in isolation displayed more intensive aggression than group-reared ones (Guhl, 1958; Gulh et al., 1960), and long-term isolation induced increased aggressive behavior in male mice (Valzelli, 1973; Vale and Montgomery, 1997). Although isolatedraising is widely used for experimental induction of agonistic behavior as mentioned above, it is not known why isolated animals increase their aggressiveness. As chickens live in groups with highly social organization (Collias et al., 1966), the disruption of contact with other chickens by isolation could be a cause of stress or mental disorder, which results in the stimulation of agonistic behavior. Matsumoto et al. (2005) have reported that isolated-raising of male mice induced many symptoms that resembled depression and anxiety, and the mice exhibited increased aggressiveness and reduced responsiveness to $\gamma$-aminobutyric acid (GABA)-A receptor agonists. As GABA-A receptor is known to play an important role in inhibition of isolation-induced aggressive behavior in mice (Puglisi-Allegra and Mandel, 1980), it is likely that isolated-raising attenuates GABA-A-mediated neurotransmission and consequently increases aggressive behavior of male animals. However, there are no previous reports which suggest the relationship between GABA neurotransmission and avian agonistic behavior until now. Sun et al. (2005) have reported that mRNA of glutamic acid decarboxylase-65 (GAD65), GABA synthesizing enzyme, was localized in the chick hypothalamus like preoptic nucleus, paraventricular nucleus, and mammillary body, which suggests the certain role of GABA in these areas. It is necessary to examine whether isolation-raising also affects GABAA-mediated neurotransmission in the brain of chickens, in the next step.

The present results showed that in the R-I test the TAF of the aggressors significantly increased from 8 to 20 days of age and their latency significantly decreased from 8 to 24 days of age. In the SI test, however, the TAF of the aggressors significantly increased and their latency significantly decreased only from 16 to 20 days of age, which showed that the expression of agonistic behavior of the aggressors in the SI test started later that in the R-I test. Rushen (1984) has reported that domestic male chicks in small mixed-sex groups show aggressive pecking in the second week of age and reach behavior levels similar to adults between the eighth and ninth weeks of life. Kruijt (1964) has also observed that the first aggressive pecking in the Burmese red jungle fowls occurs around two weeks of age, and juvenile fights start at three weeks of age. These previous reports, together with our present results, suggest that the expression of agonistic behavior of male chicks normally occurs around two weeks of age, and the R-I test advances the onset of the behavior from two weeks to one week of age by certain mechanisms. Our results also suggest that the R-I test increases aggressiveness of male chicks by stimulating their territoriality as mentioned above, which might accelerate the expression of agonistic behavior of the male chicks in the test. In the SI test, on the other hand, a pair of chicks was tested in an unfamiliar cage in which neither had established territory. File and Hyde (1978) reported that the frequency of aggressive behavior decreased when the chicks were placed in unfamiliar circumstances, which suggests that exposure to unfamiliar condition without territory formation weakens the aggressiveness of the chicks in the SI test, and consequently delays the expression of agonistic behavior compared to the R-I test.

In the present study, pecking and biting were observed in both behavioral tests, but kicking and threatening were observed only in the SI test (Table 1). In general, aggression between animals occurs in two different manners (Queiroz and Cromberg, 2006): Fights between males in order to establish territories to constitute groups (territorial behavior), and aggressive competition for hierarchy formation within groups (hierarchic dominance). The latter manner of aggression is thought to be a ritualized form of communication for the purpose of avoiding unnecessary conflict and spending less energy to establish and maintain the hierarchy within a group (Loiselet, 2004). In the SI test, a pair of chicks is put in the non-territorial space and therefore they need to communicate each other by a variety of behavioral displays under unfamiliar condition for the hierarchy determination between them. On the other hand, the R-I test induces more aggressiveness of aggressors than SI test (Fig. 3), and pecking and biting are thought to be the most effective means for chickens to attack their opponents quickly and frequently. These might be the reasons why more kinds of behavioral displays such as kicking and threatening were seen only in the SI test compared to the R-I test.

It is well-known that testosterone plays an important role in the onset of agonistic behavior of male chicks. Intramuscular administration of testosterone enanthate induces aggression of male chicks, not of female ones (Andrew, 1975; Astiningsih and Rogers, 1996), and castration of immature male chicks decreases their male-typical behaviors such as crow and aggressive fighting with other males (Quiring, 1944). In the present study, the significant differences of $\mathrm{TAF}$ and latency between the aggressors and the opponents were observed from 8 days of age in the R-I test and from 16 days of age in the SI test, respectively (Fig. 1 and 2). It is not clear, however, whether the blood concentration of testosterone rises in such immature male chicks in the R-I and SI tests. Tanabe et al. (1979) reported that the plasma concentration of testosterone in male chicks fluctuates between 100 and $300 \mathrm{pg} / \mathrm{ml}$ from 1 to 21 days of age and starts to 
increase over $400 \mathrm{pg} / \mathrm{m} l$ from 28 days of age. This suggests that plasma testosterone concentration of juvenile male chicks is relatively low and/or basal level until 21 days of age, and that this level of the hormone is enough to induce the agonistic behavior of immature male chicks. Consistent with our speculation, Soma and Wingfield (1999) reported that some of the avian species express territorial aggression in the non-breeding season when the plasma concentration of testosterone is low. Further studies are needed to evaluate the role of testosterone in the agonistic behavior of male chicks.

In conclusion, the present results suggested that the R-I test, as compared to the SI test, is a more effective tool for monitoring agonistic behavior of layer chicks. It is also suggested that the R-I test can be used for the research of brain mechanisms regulating agonistic behavior of chickens.

\section{Acknowledgments}

This work was supported by Project for the Promotion and Enhancement of the Afghan Capacity for Effective Development (PEACE) from Japan International Cooperation Agency (JICA) to S.-I. K.

\section{References}

Al-Rawi B and Craig JV. Agonistic behavior of caged chickens related to group size and area per bird. Applied Animal Ethology, 2: 69-80. 1975.

Andrew RJ. Effects of testosterone on the behaviour of the domestic chick. I. Effects present in males and not in females. Animal Behavior, 23: 139-155. 1975.

Astiningsih K and Rogers LJ. Sensitivity to testosterone varies with strain, sex, and site of action in chickens. Physiology \& Behavior, 59: 1085-1092. 1996.

Beeman EA. The effect of male hormone on aggressive behavior in mice. Physiological Zoology, 20: 373-405. 1947.

Bernhardt PC. Influences of serotonin and testosterone in aggression and dominance: Convergence with social psychology. Current Directions in Psychological Science, 6: 44-48. 1997.

Blanchard DC and Blanchard RJ. What can animal aggression research tell us about human aggression? Hormones and Behavior, 44: 171-177. 2003.

Collias NE, Collias EC, Hunsaker D and Minning L. Locality fixation mobility and social organization within an unconfined population of red jungle fowl. Animal Behaviour, 14: 550559. 1966.

de Boer SF and Koolhaas JM. 5-HT1A and 5-HT1B receptor agonists and aggression: A pharmacological challenge of the serotonin deficiency hypothesis. European Journal of Pharmacology, 526: 125-139. 2005.

File SE and Hyde JR. Can social interaction be used to measure anxiety? British Journal of Pharmacology, 62: 19-24. 1978.

Guhl AM. The development of social organization in the domestic chick. Animal Behaviour, 6: 92-111. 1958.

Guhl AM, Craig JV and Mueller CD. Selective breeding for aggressiveness in chickens. Poultry Science, 39: 970-980. 1960.

Haller J, Tóth M, Halasz J and de Boer SF. Patterns of violent aggression-induced brain c-fos expression in male mice selected for aggressiveness. Physiology \& Behavior, 88: 173182. 2006.

Hester PY. Impact of science and management on the welfare of egg laying strains of hens. Poultry Science, 84: 687-696. 2005.

Issa FA, Adamson DJ and Edwards DH. Dominance hierarchy formation in juvenile crayfish Procambarus clarkii. Journal of Experimental Biology, 202: 3497-3506. 1999.

Jones RB and Harvey S. Behavioural and adrenocortical responses of domestic chicks to systematic reductions in group size and to sequential disturbance of companions by the experimenter. Behavioural Processes, 14: 291-303. 1987.

Kjaer JB and Jørgensen H. Heart rate variability in domestic chicken lines genetically selected on feather pecking behavior. Genes, Brain, and Behavior, 10: 747-755. 2011.

Kollack-Walker S and Newman SW. Mating and agonistic behavior produce different patterns of Fos immunolabeling in the male Syrian hamster brain. Neuroscience, 66: 721-736. 1995.

Koolhaas JM, Coppens CM, de Boer SF, Buwalda B, Meerlo P and Timmermans PJ. The resident-intruder paradigm: A standardized test for aggression, violence and social stress. Journal of Visualized Experiments, 77: e4367. 2013.

Kruijt JP. Ontogeny of social behaviour in Burmese red junglefowl (Gallus gallus spadiceus) Bonnaterre. Behaviour (supplement), 12: 1-201. 1964.

Lin D, Boyle MP, Dollar P, Lee H, Lein ES, Perona P and Anderson DJ. Functional identification of an aggression locus in the mouse hypothalamus. Nature, 470: 221-226. 2011.

Loiselet J. Behaviour and feather pecking are priority areas for selection. World Poultry, 7: 22-23. 2004.

Martinez M, Phillips PJ and Herbert J. Adaptation in patterns of cfos expression in the brain associated with exposure to either single or repeated social stress in male rats. European Journal of Neuroscience, 10: 20-33. 1998.

Matsumoto K, Pinna G, Puia G, Guidotti A and Costa E. Social isolation stress-induced aggression in mice: A model to study the pharmacology of neurosteroidogenesis. Stress, 8: 85-93. 2005.

McBride $\mathrm{G}$ and Foenander F. Territorial behaviour in flocks of domestic fowls. Nature, 194: 102. 1962.

McBride G, Parer IP and Foenander F. The social organization and behaviour of the feral domestic fowl. Animal Behaviour Monographs, 2: 125-181. 1969.

Miczek KA, Maxson SC, Fish EW and Faccidomo S. Aggressive behavioral phenotypes in mice. Behavioural Brain Research, 125: 167-181. 2001.

Millman ST, Duncan IJ and Widowski TM. Male broiler breeder fowl display high levels of aggression toward females. Poultry Science, 79: 1233-1241. 2000.

Nelson RJ and Trainor BC. Neural mechanisms of aggression. Nature Reviews Neuroscience, 8: 536-546. 2007.

Panksepp J. Aggression elicited by electrical stimulation of the hypothalamus in albino rats. Physiology \& Behavior, 6: 321329. 1971 .

Puglisi-Allegra S and Mandel P. Effects of sodium n-dipropylacetate, muscimol hydrobromide and $(\mathrm{R}, \mathrm{S})$ nipecotic acid amide on isolation-induced aggressive behavior in mice. Psychopharmacology, 70: 287-290. 1980.

Queiroz SA and Cromberg VU. Aggressive behavior in the genus Gallus sp. Brazilian Journal of Poultry Science, 8: 1-14. 2006.

Quiring DP. Transplantation of testis. Bulletin of the History of Medicine, 16: 399-401. 1944.

Rushen J. Frequencies of agonistic behaviours as measures of aggression in chickens: A factor analysis. Applied Animal Behaviour Science, 12: 167-176. 1984.

Saudou F, Amara DA, Dierich A, LeMeur M, Ramboz S, Segu L, 
Buhot MC and Hen R. Enhanced aggressive behavior in mice lacking 5-HT1B receptor. Science, 265: 1875-1878. 1994.

Silverman JL, Yang M, Lord C and Crawley JN. Behavioural phenotyping assays for mouse models of autism. Nature reviews Neuroscience, 11: 490-502. 2010.

Soma K and Wingfield JC. Endocrinology of aggression in the nonbreeding season. In: Proceedings of the 22nd. International Ornithological Congress (Adams $\mathrm{N}$ and Slotow R eds.). pp. 1606-1620. BirdLife South Africa. Johannesburg. 1999.

Sun Z, Wang HB, Laverghetta A, Yamamoto K and Reiner A. The distribution and cellular localization of glutamic acid decarboxylase-65 (GAD65) mRNA in the forebrain and midbrain of domestic chick. Journal of Chemical Neuroanatomy, 29: 265-281. 2005.
Tanabe Y, Nakamura T, Fujioka K and Doi O. Production and secretion of sex steroid hormones by the testes, the ovary, and the adrenal glands of embryonic and young chickens (Gallus domesticus). General and Comparative Endocrinology, 39: 26-33. 1979.

Vale AL and Montgomery AMJ. Social interaction: Responses to chlordiazepoxide and the loss of isolation-reared effects with paired-housing. Psychopharmacology, 133: 127-132. 1997.

Valzelli L. The isolation syndrome in mice. Psychopharmacologia, 31: 305-320. 1973.

Xie J, Kuenzel WJ, Anthony NB and Jurkevich A. Subpallial and hypothalamic areas activated following sexual and agonistic encounters in male chickens. Physiology \& Behavior, 101: 344-359. 2010. 